\title{
Quarante ans d'engagement à dénoncer la "Guerre à la drogue»
}

\section{Jean Martin}

Dr med., membre de la rédaction, ancien médecin cantonal vaudois

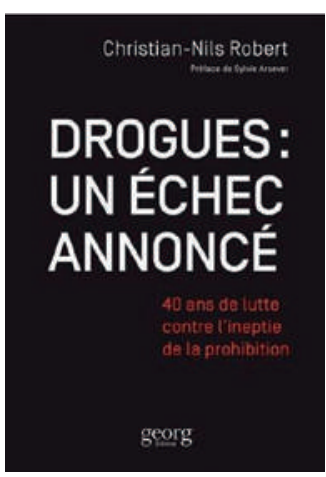

Christian-Nils Robert

Drogues: un échec annoncé - 40 ans de lutte contre l'ineptie de la prohibition

Genève: Georg Editeur; 2016.

112 pages. $25 \mathrm{CHF}$.

ISBN 978-2-8257-1045-6

Christian-Nils Robert est professeur honoraire de droit pénal à l'Université de Genève. Ce livre, qui montre son combat dès les années 1970 contre les idées reçues (pour une bonne part importées dans la foulée de la "Guerre à la drogue» décrétée par Richard Nixon), rappelle de vifs souvenirs à ceux qui ont été impliqués alors dans les questions relatives aux drogues et à la toxicomanie.

Je l'ai été depuis 1976, revenant de l'étranger après avoir pu observer la «scène» des Etats-Unis, avec entre autres la consommation à large échelle de marihuana dans la population jeune, sur les campus en particulier. Je n'étais ainsi guère prohibitionniste (ai à l'époque écrit à une dame âgée de mes proches qu'elle verrait le jour où le cannabis serait vendu en kiosque comme le tabac - et me suis donc bien trompé). La position affirmée du prof. Robert m'était néanmoins apparue très libérale... Il avait observé les dégâts qu'occasionnait la prohibition alors qu'il était directeur du Service genevois de protection de la jeunesse - prohibition renforcée en Suisse depuis un arrêt du Tribunal fédéral de 1969. Et il a écrit et dit des choses tout à fait «décoiffantes» pour la doxa de l'époque, en étant bien seul en Suisse romande si mon souvenir est bon (quelques médecins alémaniques s'engageaient dans le même sens).
A l'époque, je travaillais sous des responsables politiques aux thèses rigoristes voire punitives - et erronées (mais ma liberté de parole publique était évidemment limitée). Nombreux étaient ceux qui affirmaient vigoureusement que l'abstinence était le seul objectif qui vaut, et qui n'aimaient guère les modalités moins astreignantes de prise en charge. Mettant ainsi de côté les immenses difficultés et la souffrance de celles et ceux qui ne parvenaient pas à «s'en sortir», ou qui pour s'en sortir avaient besoin de temps. Avec l'arrivée en force du VIH/sida à la fin des années 1980, il a fallu chez nous beaucoup expliquer, voire aller à la limite de la désobéissance civile, pour faire admettre la vente libre des seringues en pharmacie, puis leur distribution gratuite. Cependant, notamment parce que la démarche avait le soutien d'un psychiatre estimé, le Dr Aldo Calanca, Vaud avait mis en place dès 1980 un programme de méthadone - qui s'est élargi avec les années. Et, progressivement, on a pu convaincre que si l'abstinence était le but idéal, il était impératif de mettre à disposition une variété de formes d'accompagnement et de traitement.

Le livre de C.-N. Robert regroupe, sans modifications, ses écrits au cours de quatre décennies sur la politique de la drogue. Le moins qu'on puisse dire est que cela «donne à réfléchir» sur la lenteur de l'évolution des attitudes et prescriptions légales; une lenteur à voir et admettre la

\section{La Guerre à la drogue a entraîné un implacable} engrenage législatif et juridique dont les conséquences paralysent encore aujourd'hui les intelligences.

réalité. Ceci dit sans préjudice à l'action pionnière de la Suisse, sous l'égide de Ruth Dreifuss surtout, dans la mise en œuvre de la politique dite des quatre piliers: prévention, répression, thérapie et réduction des risques. De manière intéressante, Robert met en parallèle de chaque article repris des citations du rapport récent de la «Global Commission on Drug Policy» [1].

A l'heure où ceux qui défendent encore une doctrine prohibitionniste qui échoue depuis un demi-siècle sont devenus plus rares, et où la Commission fédérale pour les questions liées aux drogues a pris depuis des années des positions ouvertes et éclairées, on pourrait dire que 
cet ouvrage a un caractère historique, de témoignage d'engagement. Mais il est juste, primo, de relever que Robert a eu largement raison avant les autres et, secundo, de se remémorer les funestes enchaînements et rigidités qui marquent cette histoire.

L'ouvrage bénéficie d'une préface de la journaliste Sylvie Arsever, remarquable observatrice des faits politiques et sociaux liés à la politique de la drogue. Qui dit: "Ces textes documentent non seulement le combat mené par quelques courageux obstinés, dont l'auteur, pour un peu de rationalité dans la gestion du problème, [mais] aussi un implacable engrenage législatif et juridique, dont les conséquences paralysent encore aujourd'hui les intelligences.» Elle évoque, à l'époque, la "montée en puissance d'un droit pénal tentaculaire, érigé en rempart des valeurs sociales et morales - une prolifération tumorale, une inflation punitive». Fortes paroles. Son espoir est mitigé de voir la raison s'imposer rapidement: «Les effets pervers de la prohibition, dénoncés dans les pages qui suivent, sont si apparents que peu les contestent. [Mais] l'imaginaire mondial, hanté par le martyrologue des tombés au combat contre les mafias et les cartels, continue à lier la drogue et le crime [...]. L'Assemblée générale de l'ONU consacrée à ce thème sera-t-elle celle de l'armistice? Rien n'est moins sûr avec un discours onusien jusqu'ici tétanisé par la prohibition" (les résultats de dite assemblée, tenue en avril 2016 à New York, ont en effet été très modestes).

Aussi graves et chroniques que soient les problèmes et drames liés aux drogues, il est vrai que les gouvernements sont confrontés à de multiples autres défis, dont certains (qui semblent) plus aigus. Tous nous nous sommes peu ou prou habitués à la présence des drogues et de leur usage dans nos sociétés. Il faut voir aussi que de puissants "vested interests» ont beaucoup à perdre dans une approche plus raisonnable et moins trauma-

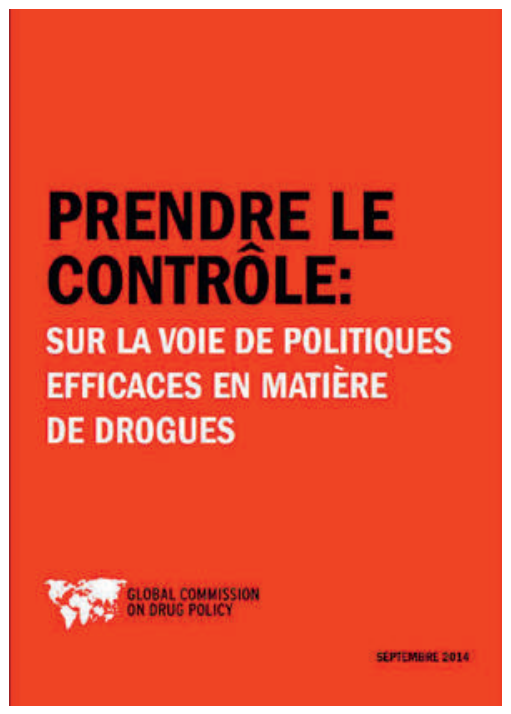

II faut donne attention au Rapport de la "Global Commis sion" de 2014 tisante pour les usagers, promouvant la règlementation plutôt que la prohibition et la criminalisation simplistes. Ces intérêts ne sont pas ceux des seuls trafiquants et mafieux, mais bien aussi liés aux énormes administrations chargées de mettre en œuvre la «Guerre à la drogue», au niveau des Nations-Unies, des gouvernements à différents niveaux et de leurs bras armés, les services de police - services perturbés d'entendre dire que leur combat n'était pas un bon combat.

L'actualité demande que soit évoquée la question du cannabis. La santé publique et le bon sens demanderaient que cesse la «chasse aux fumeurs de joints» (Ruth Dreifuss poursuit sa très estimable lutte pour que prévale la raison, et trouve «incompréhensible que le cannabis soit toujours interdit" - elle est rejointe par le policier et criminologue Olivier Guéniat [2]). Plusieurs pays tolèrent ou ont légalisé sa consommation (Portugal, Espagne, Pays-Bas, Uruguay - bientôt Canada?). Quatre Etats des USA en ont légalisé le commerce et l'usage récréatifs. En Suisse, dans les villes de Bâle, Berne, Genève et Zurich, des projets-pilotes sont envisagés, selon des modalités diverses [3]. A Lausanne, un groupe de travail a été constitué il y a plus d'un an, après qu'ait pu être levée la non-entrée en matière des partis de droite. Dans un utile nouveau rapport publié le 14 juin, Addiction Suisse présente une vue d'ensemble de la situation et discute les développements possibles.

En ce qui concerne l'usage médical du cannabis, il est accepté dans de nombreux pays. Chez nous, il est encore soumis à des exigences restrictives, alors que son emploi en clinique devrait être admis de routine plutôt que comme exception - ce que demandent des confrères neurologues notamment.

Revenant au livre de C.-N. Robert, on voit qu'il illustre les efforts au cours du temps de quelques "Querdenker» (francs-tireurs) qui ont posé très tôt, sans être entendus, de très bonnes questions. Aujourd'hui encore, c'est un défi de faire entendre et de faire comprendre que ce n'est pas dans des démarches extrémistes (dans un sens ou dans l'autre, d'ailleurs) qu'on avancera, mais bien en élaborant et testant des modèles qui tiennent compte de la réalité des échecs et de l'expérience acquise. Ne pas persévérer dans les impasses, oser mettre au panier les idées reçues et les tabous.

\section{Références}

1 Global Commission on Drug Policy. Rapport «Prendre le contrôle: sur la voie de politiques efficaces en matière de drogues», 2014

2 Le cannabis est moins nocif que l'alcool (interview d'O. Guéniat par C. Zünd). Le Temps, 23 mars 2016.

3 Fumer un joint légalement. 24 heures et Tribune de Genève, 23 juin 2016. Voir aussi le dossier publié par L’Hebdo le 2 juin 2016. Toutefois, la mise en œuvre de tels projets reste incertaine, la vieille garde dogmatique ne désarme pas: la conseillère nationale UDC V. Herzog a déposé une initiative visant à limiter les dérogations que peut accorder l'OFSP pour des recherches liées aux drogues. 